INPLASY

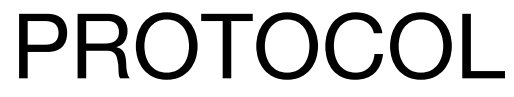

To cite: Chen et al. A new therapy for chronic heart failure for using acupuncture: a systematic review and metaanalysis of randomised controlled trials. Inplasy protocol 202040005. doi: 10.37766/inplasy2020.4.0005

Received: 02 April 2020

Published: 02 April 2020

Corresponding author: Deyu Fu

fdy65@126.com

Author Affiliation:

ShanghaiUniversity of traditional Chinese Medicine

Support: None

Review Stage at time of this submission: The review has not yet started.

Conflicts of interest: None.

\section{A new therapy for chronic heart failure for using acupuncture: a systematic review and meta-analysis of randomised controlled trials}

Chen, X1; Fan, X²; Shi, X33 Yao, L4; Fu, D5.

\begin{abstract}
Objective: To know whether acupuncture therapy is effective and safe for treating chronic heart failure.

Condition being studied: Chronic heart failure (CHF) is a progressive syndrome that results in a poor quality of life for the patient and places an economic burden on the health care system. Optimal therapy for CHF involves identification and correction of potentially reversible precipitants, target-dose titration of medical therapy, and management of hospitalizations for compensation. Thus, it is of great importance for the prevention and treatment of the disease. Consequently, there is an urgent need for innovative approaches and strategies for treatment. Acupuncture therapy for CHF has been widely used in clinical applications, considered that it has the advantages of simple and safe operation without obvious adverse reaction, and can significantly improve the quality of the life. However, there has no study evaluate the benefits of acupuncture therapy for patient with CHF.
\end{abstract}

INPLASY registration number: This protocol was registered with the International Platform of Registered Systematic Review and Meta-Analysis Protocols (INPLASY) on 02 April 2020 and was last updated on 02 April 2020 (registration number INPLASY202040005.

\section{INTRODUCTION}

Objectives / Review question: To know whether acupuncture therapy is effective and safe for treating chronic heart failure.
Condition being studied: Chronic heart failure (CHF) is a progressive syndrome that results in a poor quality of life for the patient and places an economic burden on the health care system. Optimal therapy for CHF involves identification and correction 
of potentially reversible precipitants, target-dose titration of medical therapy, and management of hospitalizations for compensation. Thus, it is of great importance for the prevention and treatment of the disease. Consequently, there is an urgent need for innovative approaches and strategies for treatment. Acupuncture therapy for CHF has been widely used in clinical applications, considered that it has the advantages of simple and safe operation without obvious adverse reaction, and can significantly improve the quality of the life. However, there has no study evaluate the benefits of acupuncture therapy for patient with CHF..

\section{METHODS}

Participant or population: Patients diagnosed as Chronic Heart Failure (CHF) with no limitations on gender, age, course and co-morbidity will be included. But baseline characteristics of each study should be consistent.

Intervention: The form of acupuncture should be rooted in traditional meridian theory, which means auricular acupuncture, scalp acupuncture, transcutaneous electrical nerve stimulation, laser acupuncture and wristankle acupuncture will be excluded. The stimulating modalities can be filiform acupuncture, electro-acupuncture, fire needle acupuncture, plum-blossom needle acupuncture, acupoint catgut implantation, acupoint injection, acupoint drug application, acupressure, or acupoint moxibustion.

Comparator: Control groups are treated with any type of conventional therapy for Chronic Heart Failure (CHF) without acupuncture.

Study designs to be included: Inclusion criteria: RCTs. Exclusion criteria: NonRCTs.

Eligibility criteria: Adults with chronic heart failure treated by both manual acupuncture and electro-acupuncture.
Information sources: A comprehensive search of 7 medical databases, including PubMed, EMBASE (OVID), the Cochrane Library, China National Knowledge Infrastructure (CNKI), Chinese Scientific Journals Database (VIP), Wanfang Database and Chinese Biomedical Database (CBM) through January 2020 will be conducted without language restriction.

Main outcome(s): Clinical outcomes will be eligible, including left ventricular ejection fraction (LVEF), BNP, NT-proBNP, quality of life score, et al.

Additional outcomes: Depression (measured by the 9-item Patient Health Questionnaire), anxiety (measured by the 7item Generalized Anxiety Disorder Questionnaire), overall symptom distress (measured by the General Symptom Distress Scale), specific symptoms (pain, fatigue, and shortness of breath), number of hospitalizations, and mortality.

Data management: Review Manager5.3 and StataMP 14.

Quality assessment / Risk of bias analysis: Risk of bias (ROB) assessment was conducted by two independent authors. In the event of a disagreement while extracting data or assessing ROB, the third author resolved the discrepancy. The domains of ROB assessment are random sequence generation, allocation concealment, participant and personnel blinding, outcome assessment blinding, incomplete data, selective reporting and other biases according to the Cochrane handbook for systematic reviews. ROB was graded as high, low, or unclear. When we evaluated participant and personnel blinding, we graded ROB as low even though only participants were blinded, as the practitioner cannot be blinded in an acupuncture trial.

Strategy of data synthesis: Dichotomous data will be calculated by risk ratio (RR), while continuous data by mean difference (MD), with $95 \%$ confidence intervals. 
Search strategy: (Acupuncture OR electroacupuncture OR 'acupuncture therapy' OR ear acupuncture) AND (Heart failure $O R$ cardiac failure $O R$ myocardial failure OR heart insufficiency OR cardiac insufficiency OR cardiomyopathies OR ventricular dysfunction).

Subgroup analysis: Subgroup analysis defined by NYHA heart, gender, type of acupuncture, severity of condition, or intervention period, and sensitivity analysis stratified by quality of included trials will be performed to detect heterogeneity or publication bias if sufficient sources are available.

Sensibility analysis: If sensibility analysis is necessary, we will do it.

Language: English and Chinese.

Keywords: acupuncture, Chronic heart failure,meta-analysis.

Contributions of each author:

Author 1 - Literature Search; Data Collection and Quality assessment;Statistical Analysis;Write the article.

Author 2 - Literature Search; Data Collection and Quality assessment;Statistical Analysis;Write the article.

Author 3 - Literature Search; Data Collection and Quality assessment;Statistical Analysis;Write the article.

Author 4 - Literature Search; Data Collection and Quality assessment;Statistical Analysis;Write the article. 